\title{
Mid-infrared resonant ablation for selective patterning of thin organic films
}

\author{
Sanjeev Naithani $^{1 \mathrm{a}}$, Charles Duterte ${ }^{\mathrm{b}}$, Marieta Levichkova ${ }^{\mathrm{c}}$, Arnaud Grisard ${ }^{\mathrm{d}}$, David Schaubroeck ${ }^{\mathrm{a}}$, \\ Eric Lallier ${ }^{\mathrm{d}}$, Yves Hernandez ${ }^{\mathrm{b}}$, Karsten Walzer ${ }^{\mathrm{c}}$, Geert Van Steenberge ${ }^{\mathrm{a}}$ \\ ${ }^{\mathrm{a} C e n t r e}$ for Microsystems Technology (CMST), imec and Ghent University, Technologiepark 914, \\ 9052 Gent, Belgium. \\ ${ }^{\mathrm{b}}$ Multitel, Rue Pierre et Marie Curie 2, 7000 Mons, Belgium. \\ ${ }^{c}$ Heliatek GmbH, Treidlerstraße 3, 01139 Dresden, Germany. \\ ${ }^{\mathrm{d}}$ Thales Research \& Technology (TRT), Av. Augustin Fresnel 1, 91767 Palaiseau cedex, France.
}

\begin{abstract}
The fast growing market of organic electronics, including organic photovoltaics (OPV), stimulates the development of versatile technologies for structuring thin-film materials. Ultraviolet lasers have proven their full potential for patterning single organic layers, but in a multilayer organic device the obtained layer selectivity is limited as all organic layers show high UV absorption. In this paper, we introduce mid-infrared (IR) resonant ablation as an alternative approach, in which a short pulse mid-infrared laser can be wavelength tuned to one of the molecular vibrational transitions of the organic material to be ablated. As a result, the technique is selective in respect of processing a diversity of organics, which usually have different infrared absorption bands. Mid-IR resonant ablation is demonstrated for a variety of organic thin films, employing both nanosecond ( $15 \mathrm{~ns})$ and picosecond $(250 \mathrm{ps})$ laser pulses tunable between 3 and 4 microns. The nanosecond experimental set-up is based on a commercial laser at $1064 \mathrm{~nm}$ pumping a singly resonant Optical Parametric Oscillator (OPO) built around a Periodically-Poled Lithium Niobate (PPLN) crystal with several Quasi-Phase Matching (QPM) periods, delivering more than $0.3 \mathrm{~W}$ of mid-IR power, corresponding to $15 \mu \mathrm{J}$ pulses. The picosecond laser set-up is based on Optical Parametric Amplification (OPA) in a similar crystal, allowing for a comparison between both pulse length regimes.
\end{abstract}

The wavelength of the mid-infrared laser can be tuned to one of the molecular vibrational transitions of the organic material to be ablated. For that reason, the IR absorption spectra of the organic materials used in a typical OPV device were characterized in the wavelength region that can be reached by the laser setups. Focus was on OPV substrate materials, transparent conductive materials, hole transport materials, and absorber materials. The process has been successfully demonstrated for selective thin film patterning, and the influence of the various laser parameters is discussed.

Keywords: laser patterning, mid-infrared, OPV layers, resonant ablation, thin films

\section{INTRODUCTION}

Organic and printed electronics is a platform technology based on the combination of new materials and cost-effective, large area production processes that open up new fields of application. Thin, light-weight, flexible and environmentally friendly processes and products are some of the key advantages organic and printed electronics can offer. It also enables a wide range of electrical components that can be produced and directly integrated in low cost roll-to-roll processes. Intelligent packaging, low cost RFID (radio-frequency identification) transponders, rollable displays, flexible solar cells, disposable diagnostic devices or games, and printed batteries are just a few examples of promising fields of application [1][2].

The technology relies on thin films of electrically active materials that can be used as conductors, semiconductors, luminescent, electrochromic or electrophoretic materials. There are many approaches on the material side and the resulting questions - organic or inorganic, solution based or evaporated - are still under discussion. Transparent organic

${ }^{1}$ sanjeev.naithani@ugent.be; phone +32-9 264 5512; fax +32-9 264 5374; www.ugent.be

Laser Sources and Applications II, edited by Jacob I. Mackenzie, Helena Jelĺnková, Takunori Taira, Marwan Abdou Ahmed, Proc. of SPIE Vol. 9135, 91350K · C 2014 SPIE · CCC code: 0277-786X/14/\$18 · doi: 10.1117/12.2052763 
conductors are picking up in performance but still show inferior conductivities in comparison to metal oxides like ITO. Advantageously the polymers allow for wet processing and the flexibility of the polymer coatings makes them the chosen candidates for the replacement of (brittle) inorganic materials. Continued progress in increasing the electrical conductivity of PEDOT:PSS polymer has been achieved over the past years [3]. Most of the organic semiconductors used today are p-type (like pentacene, polythiophene and TIPS-pentacene), but more n-type materials are becoming available (like NTDI, PTCDI, and Polyera's PDI compounds); combined with the p-type materials and matching dielectrics, these open the door to CMOS-circuits. For organic photovoltaic (OPV) materials, there has been rapid progress in the power conversion efficiency of organic active-layer donor materials, now achieving efficiencies as high as $12.0 \%$ [4], combining two absorber materials, which convert light of different wavelengths. However, significant work still remains to translate small-area, lab-scale cell performance into the large-area, production-level modules required to address market needs.

A wide range of large area deposition and patterning techniques can be used for organic and printed electronics. However, this paper describes the use of laser technology for patterning organic photovoltaic thin film materials. By laser patterning, very fine feature sizes can be obtained with high process speeds from homogeneously deposited layer sequences. Proper power density adjustment, wavelength and pulse width selection of the laser beam have to be utilized for selective removal of different thin layers. Ultraviolet lasers have proven their full potential for patterning single organic layers, but in a multilayer organic device the obtained layer selectivity is limited as all organic layers show high UV absorption. We introduce mid-infrared resonant ablation as an alternative approach, in which a short pulse midinfrared laser can be wavelength tuned to one of the molecular vibrational transitions of the organic layer to be ablated. In this wavelength region, the absorption of the material is linked to vibrational transitions of the molecular chain rather than resonant electronic transitions. As a result, the technique is selective in respect of processing a diversity of organic materials, which usually have different infrared absorption bands.

Mid-infrared lasers have already been tested and validated for material processing. Most of the studies [5] employing long wavelength $\mathrm{CO}_{2}$ lasers revealed that the chemical structures and compositions of the ablated polymers were strongly dependent on the wavelength of ablation. In most of these experiments the laser-pulse duration was in the range of a few hundreds of nanoseconds. More recent works employed a Free Electron Laser (FEL) for studying the wavelength influence on polymer Pulsed Laser Deposition (PLD) over a large infrared region. After evaluating the position and intensity of the vibrational absorption bands of the polymers in the IT spectrum, the laser wavelength can be matched to a maximum of absorption of the polymer target. The results show that PolyStyrene (PS) has relatively high absorption coefficients in some specific IR absorption bands e.g. at $3.31 \mu \mathrm{m}$, and at $3.43 \mu \mathrm{m}$, corresponding to aromatic ring $\mathrm{C}-\mathrm{H}$ stretching modes, and aliphatic $\mathrm{C}-\mathrm{H}$ asymmetric stretching modes, respectively. However, in most applications, FELs are not practical since they are coupled to huge and expensive accelerators. Therefore, new technologies are being developed to replace FELs with bench-top solid-state photonic sources [6][7].

In our previous study [8], resonant ablation of Polymethylmethacrylate) (PMMA) on glass has been successfully demonstrated. In this paper, we demonstrate mid-IR resonant ablation for a variety of organic thin-films, employing both nanosecond ( $15 \mathrm{~ns})$ and picosecond ( $250 \mathrm{ps)}$ laser pulses tunable between 3 and 4 microns. The nanosecond experimental set-up is based on a commercial laser at $1064 \mathrm{~nm}$ pumping a singly resonant Optical Parametric Oscillator (OPO) built around a Periodically-Poled Lithium Niobate (PPLN) crystal with several Quasi-Phase Matching (QPM) periods, delivering more than $0.3 \mathrm{~W}$ of mid-IR power, corresponding to $15 \mu \mathrm{J}$ pulses. The picosecond laser set-up is based on Optical Parametric Amplification (OPA) in a similar crystal, allowing for a direct comparison between both pulse length regimes.

The wavelength of the mid-infrared laser can be tuned to one of the molecular vibrational transitions of the organic material to be ablated. For that reason, the IR absorption spectra of the organic materials used in a typical organic photovoltaic device were characterized in the wavelength region that can be reached by the laser setups. Focus was on three materials prototypical for organic solar cells: as a planarization layer the polymer PEDOT:PSS (poly(3,4ethylenedioxythiophene) poly(styrenesulfonate)); as hole transport layer two typical materials, that can be used alternatively in OSCs: BPAPF (9,9-bis[4-(N,N-bis-biphenyl-4-yl-amino)phenyl]-9H-fluorene) and $\alpha$-NPB (N,N'diphenyl-N,N'-bis(1-naphthyl)-1,1'-biphenyl-4,4"-diamine), and as a typical high-efficiency absorber materials we selected Heliatek's HDR014 (an oligothiophene derivative with non-disclosed structure), and DCV4T-Et2 (2,2'-[(3,3"'diethyl[2,2':5':2":5",2"'-quaterthiophene]-5,5"'-diyl)dimethylidyne]bis- Propanedinitrile)[9]. BPAPF and $\alpha$-NPB are widely used as hole transport materials in organic solar cells and organic light-emitting diodes[10][11]. HDR014 is a 
donor material with strong absorption in the red spectral region. The process has been successfully demonstrated for selective thin film patterning, and the influence of the various laser parameters is discussed.

\section{EXPERIMENTAL WORK}

\subsection{Sample preparation}

Thin films of PEDOT:PSS (poly(3,4-ethylenedioxythiophene) poly(styrenesulfonate)) on both glass (Prinz Optics) and PET (polyethylene terephthalate) (DuPont) substrates with nominal thicknesses of 70, 140, and $210 \mathrm{~nm}$ were produced via spin-coating. PEDOT:PSS layers were produced using a commercial aqueous solution (Heraeus) with a PEDOT:PSS ratio of 1:6 (by weight) and solid content of 1.3 to $1.7 \%$. The nominal thickness of the PEDOT:PSS layer was reached after a multi-step spin-coating process. The thickness of the layers after a single step at rotation speed of $1500 \mathrm{rpm}$ and acceleration of $500 \mathrm{rpm} / \mathrm{s}$ corresponds to about $70 \mathrm{~nm}$. After each coating step, the PEDOT:PSS layers were annealed for 15 minutes at $120^{\circ} \mathrm{C}$ to remove the solvent.

BPAPF, $\alpha$-NPB, DCV4T-Et2 and HDR014 films with nominal thickness of $140 \mathrm{~nm}$ were deposited on glass substrates (Prinz Optics or Glas-Meyer) via thermal evaporation in a UHV chamber (Kurt J. Lesker) at a deposition rate of $0.3 \AA / s$. HDR014, DCV4T-Et2 (both Heliatek) and BPAPF (Lumtec) were purified by vacuum sublimation prior to thin film preparation. The hole transport material $\alpha$-NPB (Sensient) was used as synthesized. The film thickness was controlled by quartz crystal microbalance (QCM).

\subsection{Mid-infrared absorption spectra}

The IR absorption spectra of the prototypical materials used in an OPV stack were obtained in the wavelength regions of interest. Attenuated total reflectance infrared spectroscopy (ATR-IR) was employed to characterize the substrate foils, since a measurement in transmission requires a sample thickness limited to a few tens of microns. The thin-film materials were deposited on an IR transparent substrate material (e.g. $\mathrm{CaF}_{2}$ ), to avoid overlap in the spectra of thin-film coating versus substrate material. Reliable spectra could be obtained using measurements in transmission and in reflection. Further, powder spectra in $\mathrm{KBr}$ pellets were collected for comparison with thin-film measurements.

\subsection{Laser processing set up}

The mid-IR ablation experiments were performed using two set-ups, providing nanosecond and picosecond pulses. The nanosecond laser is based on a commercial laser at $1064 \mathrm{~nm}$ pumping with $\sim 15$ nanosecond pulses at a repetition rate of $20 \mathrm{kHz}$. A singly resonant Optical Parametric Oscillator (OPO) build around a PPLN crystal with several Quasi-Phase Matching (QPM) periods was used in this configuration [12]. In the OPO cavity, the signal can oscillate between 1500 $\mathrm{nm}$ and $1650 \mathrm{~nm}$ wavelengths, corresponding to idler wavelengths from 3660 to $2996 \mathrm{~nm}$. Coarse tuning can be obtained by translating the PPLN crystal, thus changing the QPM period. In addition, a wavelength fine tuning is achieved by heating the crystal, which allows us to achieve continuous wavelength tuning between $3000 \mathrm{~nm}$ and $3600 \mathrm{~nm}$. This means that by combining coarse and fine wavelength tuning, it is possible to address the IR absorption bands in the range of 3 to $4 \mu \mathrm{m}$. The picture of the nanosecond laser patterning set-up is shown in Figure 1(below).

After the OPO and filtering section (designated to remove residual pump and signal), the laser beam is loosely collimated by a Si plano-convex lens (100 $\mathrm{mm}$ focal length), and focused by a mid-IR microscope objective (6 mm focal length) on the sample. The samples were mounted on an automatic translational stage to allow for a controlled amount of laser shots, a key parameter in thin-film patterning.

During the experiments, the influence of scanning speed, pulse energy and mid-IR wavelength was studied. Scanning speeds were widely varied from $0.01 \mathrm{~mm} / \mathrm{s}$ to $300 \mathrm{~mm} / \mathrm{s}$, pulse energies were adjusted by varying the power (attenuator unit) while keeping the pulse repetition rate fixed at $20 \mathrm{kHz}$. The average power was varied from $120 \mathrm{~mW}$ to $170 \mathrm{~mW}$, resulting in corresponding pulse energies of $6 \mu \mathrm{J}$ to $8.5 \mu \mathrm{J}$. All thin-film organic materials were investigated for two wavelengths, allowing for a comparison of resonant versus non-resonant absorption. The signal wavelengths (around $1500 \mathrm{~nm}$ ) were measured by an optical spectrum analyzer (OSA), whereas the corresponding idler wavelengths (around $3 \mu \mathrm{m})$ were calculated. 


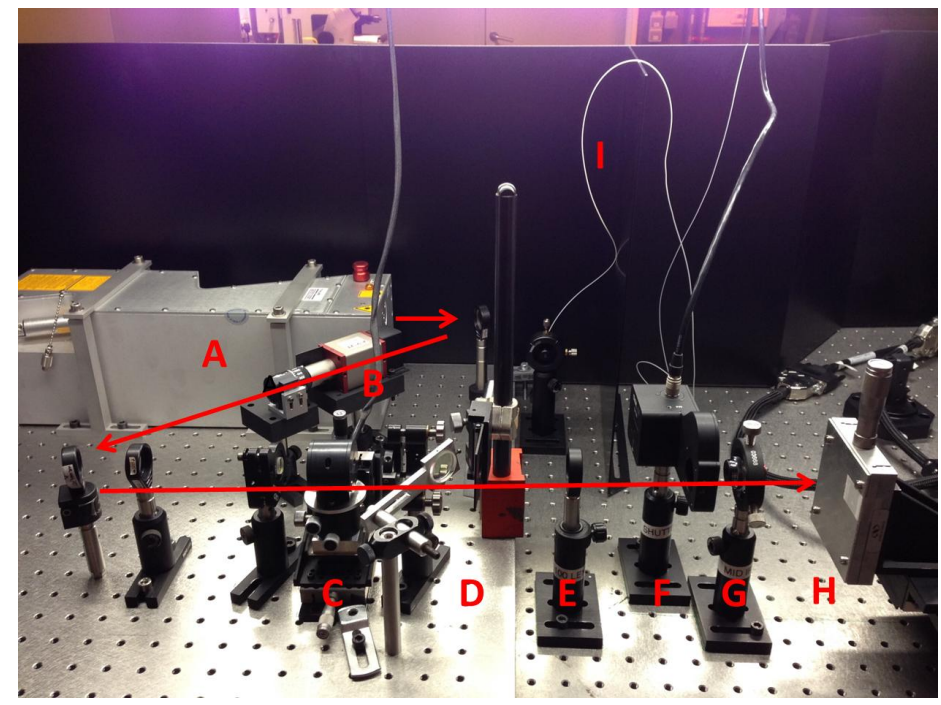

Figure 1 Picture of the nanosecond laser set-up: A - Laser, B - Isolator / attenuator unit, C - PPLN crystal / OPO section, D Filtering section, E - Collimating lens, F - Shutter, G - mid-IR objective, H - Sample / translational stages, I - Coupling fiber to OSA.

On the other hand, the picosecond laser set-up is based on a commercial laser (Amplitude Systems, France) emitting at $1028 \mathrm{~nm}$ and modified to have $250 \mathrm{ps}$ pulse duration at the output. The repetition rate has been fixed to $25 \mathrm{kHz}$ and the maximum power at $4 \mathrm{~W}$ corresponds to pulse energy of $160 \mu \mathrm{J}$. A multi-grating PPLN crystal has been used for the experiments in a double pass OPA configuration [13]. The signal source (CW, $5 \mathrm{~mW})$ to be amplified is tunable between $1460 \mathrm{~nm}$ and $1580 \mathrm{~nm}$ making possible to have an idler between $2.94 \mu \mathrm{m}$ and $3.47 \mu \mathrm{m}$. The maximum output power obtained at $3.03 \mu \mathrm{m}$ and $3.37 \mu \mathrm{m}$ is $80 \mathrm{~mW}$ and $160 \mathrm{~mW}$ respectively (see results on mid-IR spectra as a motivation for these two particular wavelengths). An aspherical lens has been used to focus the beam on the sample to process. The $\mathrm{M}^{2}$ of the beam is unknown but for a perfect beam $\left(\mathrm{M}^{2}=1\right)$ the spot diameter is estimated to be around $12 \mu \mathrm{m}$ in the focal plane. The schematic diagram of the picosecond laser set-up is shown in the Figure 2.

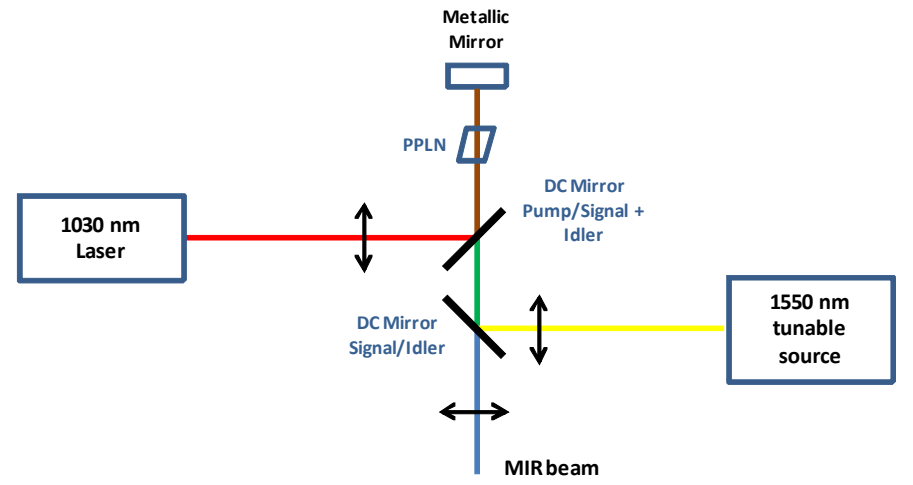

Figure 2 Schematic diagram of the picosecond laser set up.

\subsection{Analysis}

The investigation and analysis of the samples after experiments were performed by using an optical microscope and a non-contact optical profiler (WYKO NT3300). Scanning electron microscopy (SEM) was used to investigate the surface topography and possible contamination after the ablation experiments. SEM analysis was performed on a JEOL-5600 and a Zeiss Neon 40 instruments. Prior to analysis, the samples were coated with thin gold layer (roughly $20 \mathrm{~nm}$ ) via plasma magnetron sputter coating. For elemental surface analysis, energy-dispersive X-ray spectroscopy (EDX) was carried out to get spatially highly resolved elemental surface mapping. It turned out that this technique is a very efficient tool to characterize the quality of laser scribes. 


\section{RESULTS AND DISCUSSION}

\subsection{PEDOT:PSS mid-IR laser patterning}

In the first part of the investigation, we evaluated the use of a long wavelength mid-IR laser (nanosecond) for ablation of PEDOT:PSS thin organic films. Various layers of PEDOT:PSS were prepared using the procedure described in the experimental part. To study the influence of overlap between the absorption of the PEDOT:PSS thin-film and the substrate, both glass and PET foil carriers were selected. The absorption spectra of PEDOT:PSS and PET are shown in Figure 3.

From these spectra, it is observed that PEDOT:PSS has a two absorption bands around 3.4 and $3.5 \mu \mathrm{m}$, and a second broader absorption band at $3.03 \mu \mathrm{m}$. The latter can be ascribed to the O-H stretch of PSS-H. The band around $3.4 \mu \mathrm{m}$ can be assigned to the asymmetric aliphatic $\mathrm{C}-\mathrm{H}$ stretch of the PSS units. The other band around $3.5 \mu \mathrm{m}$ arises from the symmetric aliphatic C-H stretch of PSS. Note that the PEDOT:PSS weight ratio is 1:6 in this case. PET has absorption bands at $2.91 \mu \mathrm{m}$, and around $3.37 \mu \mathrm{m}$. The band around $2.9 \mu \mathrm{m}$ arises from the $\mathrm{O}-\mathrm{H}$ stretch of the PET functional end groups. The band at $3.37 \mu \mathrm{m}$ can be assigned to the asymmetric $\mathrm{C}-\mathrm{H}$ stretch of $\mathrm{CH}_{2}$ moieties. Already now it is clear from the overlap in spectra between PEDOT:PSS and PET, that for selective removal of PEDOT:PSS from PET, the absorption band around 3.4-3.5 $\mu \mathrm{m}$ has to be avoided, because of cross-sensitivity with the PET substrate. On the other hand, a wavelength of $3.03 \mu \mathrm{m}$, could be a good candidate.
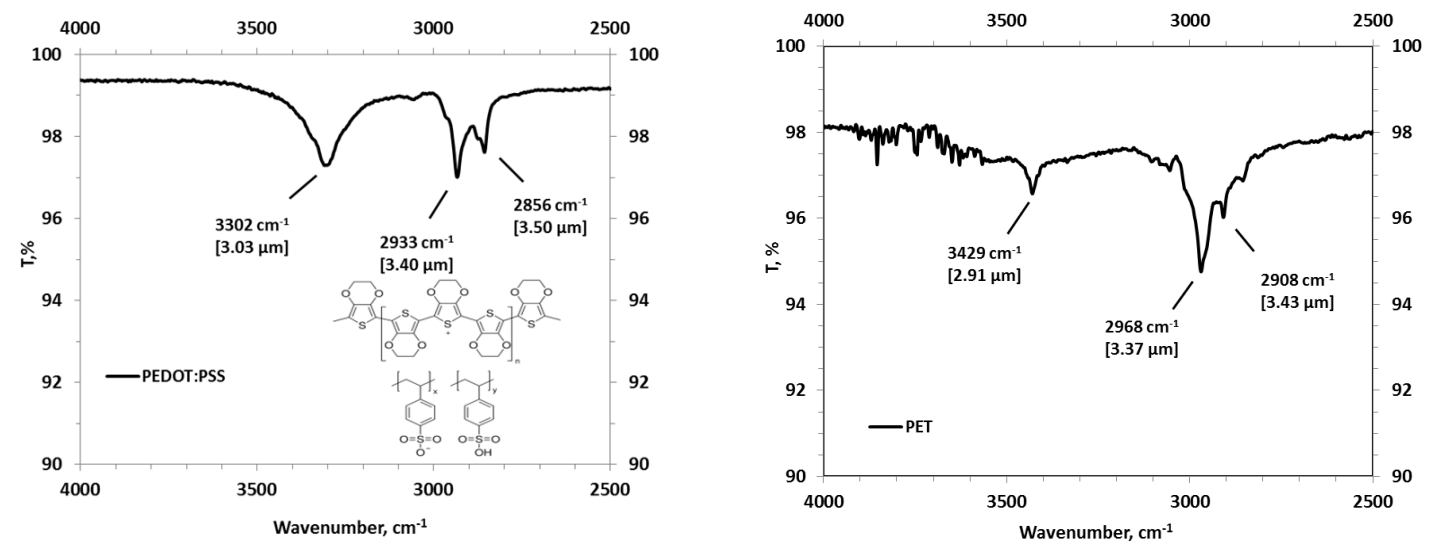

Figure 3 PEDOT:PSS (left) and PET (right) infrared spectra in the wavelength region that can be reached by the mid-IR laser set-ups.

The initial goal of these experiments was to prove that a mid-IR laser could be used for removing a thin film of $140 \mathrm{~nm}$ in thickness, using a glass carrier. Two wavelengths were investigated: $3.40 \mu \mathrm{m}$, corresponding to resonant absorption, and $3.30 \mu \mathrm{m}$, corresponding to non-resonant absorption. The (nanosecond) laser parameters used during these experiments are $160 \mathrm{~mW}$ of power, $20 \mathrm{kHz}$ pulse repetition rate and various scan speeds. Removal of the PEDOT:PSS film was demonstrated for resonant ablation $(3.40 \mu \mathrm{m})$, for which the optical microscope image and SEM micrograph are shown in Figure 4. 

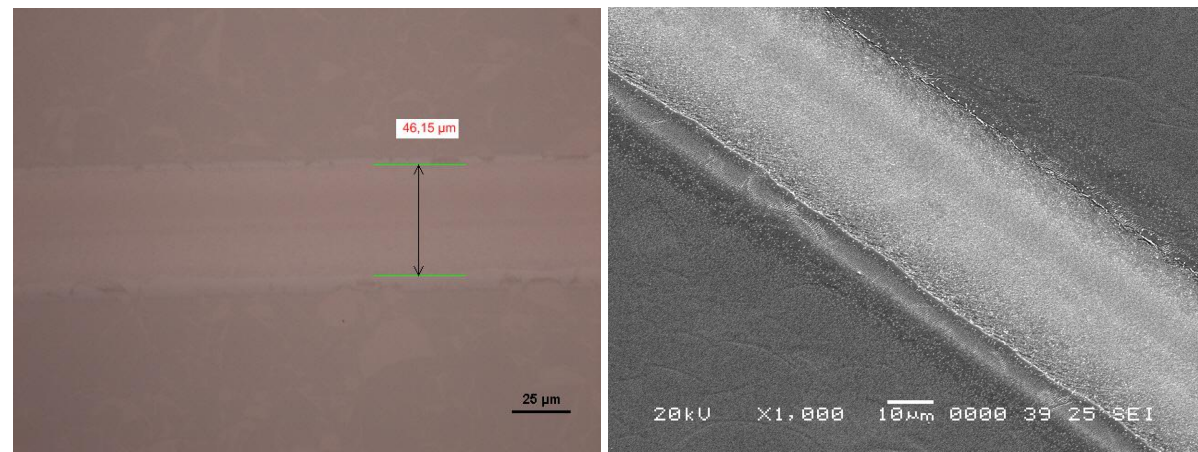

Figure 4 PEDOT:PSS patterning on glass, using resonant ablation at $3.40 \mu \mathrm{m}$. Optical microscopic image (left) and SEM micrograph (right).
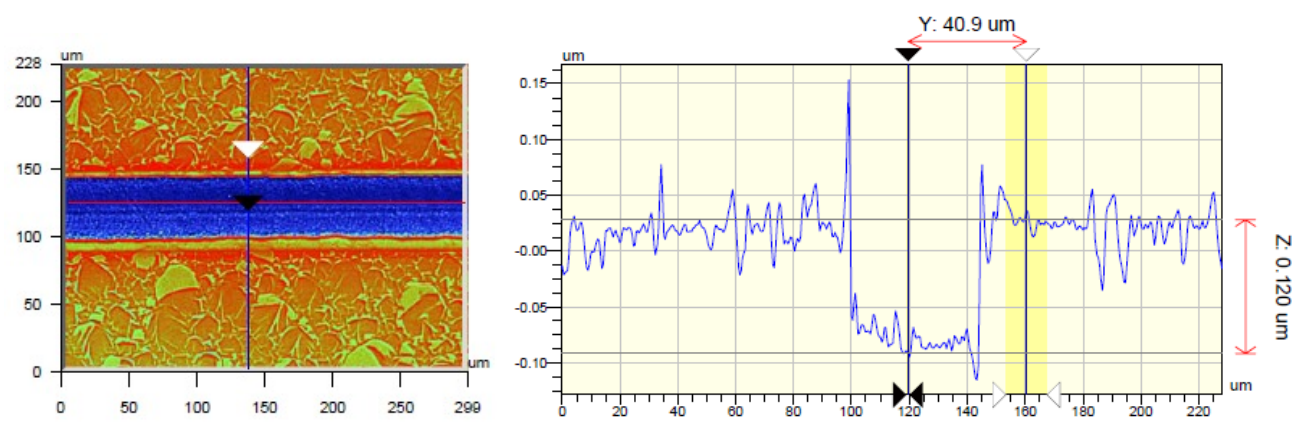

Figure 5 Depth profile measurement (non-contact optical profiler) after mid-IR laser ablation of PEDOT:PSS (specified layer thickness of $140 \mathrm{~nm}$ ).

In addition to visual inspection, the depth profile of the ablated track has been measured using a non-contact optical profiler and the result is shown in Figure 5 (above). Apart from the fact that the surface roughness of the PEDOT:PSS film seems to be high, the measured depth of the ablated crater is about $120 \mathrm{~nm}$, well corresponding to the specified layer thickness of $140 \mathrm{~nm}$, so we could assume that the complete organic layer has been removed (although this was not verified by more detailed surface analysis). The spike at the left edge of the ablated groove is attributed to a measurement artifact of the optical profiler. Hence, mid-IR resonant ablation has been shown for thin organic films. Resonant ablation at the absorption peak of $3.4 \mu \mathrm{m}$ removal of PEDOT:PSS thin film was possible, although the scan speed is very low.

In contrast, for similar experimental conditions, but employing a non-resonant wavelength $(3.30 \mu \mathrm{m})$ no PEDOT:PSS removal was observed. To visualize the influence of the mid-IR wavelength on material removal, a PEDOT:PSS film was coated on a PET substrate, and the (picosecond) laser wavelength was tuned between 3.41 and $3.50 \mu \mathrm{m}$. Note that in this case the absorption spectra of PEDOT:PSS and PET have to be taken into account (Figure 3). It is clear from the experimental results shown in Figure 6, that the influence of changing the wavelength by $100 \mathrm{~nm}$ is significant: ablating a few microns compared to a few hundreds of nanometers. The explanation can be found in the much stronger absorption of the PET substrate in the case of $3.41 \mu \mathrm{m}$, compared to $3.50 \mu \mathrm{m}$. 

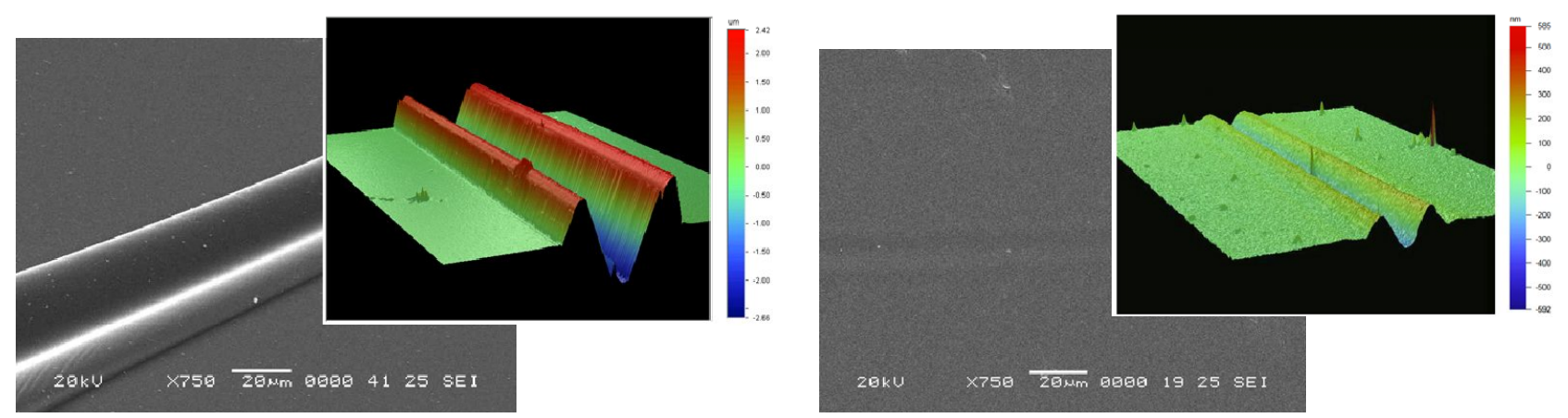

Figure 6 SEM micrographs and profile measurements, for identical experimental laser settings $(80 \mathrm{~mW}-40 \mathrm{~mm} / \mathrm{s}-2 \mu \mathrm{J})$, except for the wavelength: $3.41 \mu \mathrm{m}$ (left) and $3.50 \mu \mathrm{m}$ (right).

\subsection{Picosecond laser patterning of thin organic films on PET foil}

In the first stage of experiments, PEDOT:PSS removal on PET with a picosecond laser was performed. The thickness of PEDOT:PSS in this case was $210 \mathrm{~nm}$. In these tests the mid-IR radiation was generated by an OPG configuration leading to about $8 \mu \mathrm{J}$ per pulse at $3.03 \mu \mathrm{m}$. With a repetition rate of $25 \mathrm{kHz}$ and a scanning speed of $90 \mathrm{~mm} / \mathrm{s}$, the scribing of the PEDOT:PSS layer could be achieved, as depicted in Figure 7.

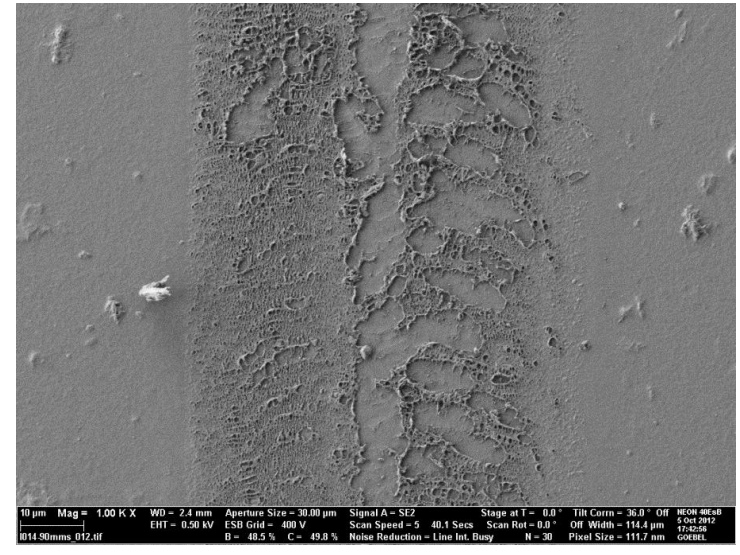

(a)

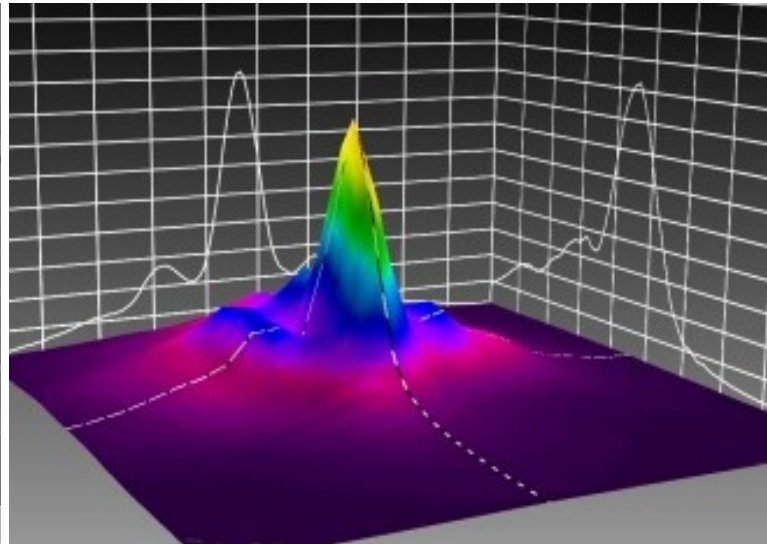

(b)

Figure 7 (a) SEM micrograph after picosecond laser ablation $(3.03 \mu \mathrm{m})$ of $210 \mathrm{~nm}$ PEDOT:PSS on PET. Laser parameters: $90 \mathrm{~mm} / \mathrm{s}$, pulse energy $\sim 8 \mu \mathrm{J}$. (b) beam profile of the mid-IR source used for this experiment.

As one can deduce from the previous pictures, the quality of the scribes strongly depends on the beam quality of the midIR source. In order to improve the results, the source was changed to an OPG /OPA configuration and the output beam was filtered with diaphragm before being focused on the sample.

In this configuration, the quality of the scribe was better as shown in Figure 8 (obtained with $3 \mu \mathrm{J}$ per pulse at $100 \mathrm{~mm} / \mathrm{s}$ ) although, as we do not have a top-hat configuration at this wavelength, the complete removal of the material between each pulse was not possible. 


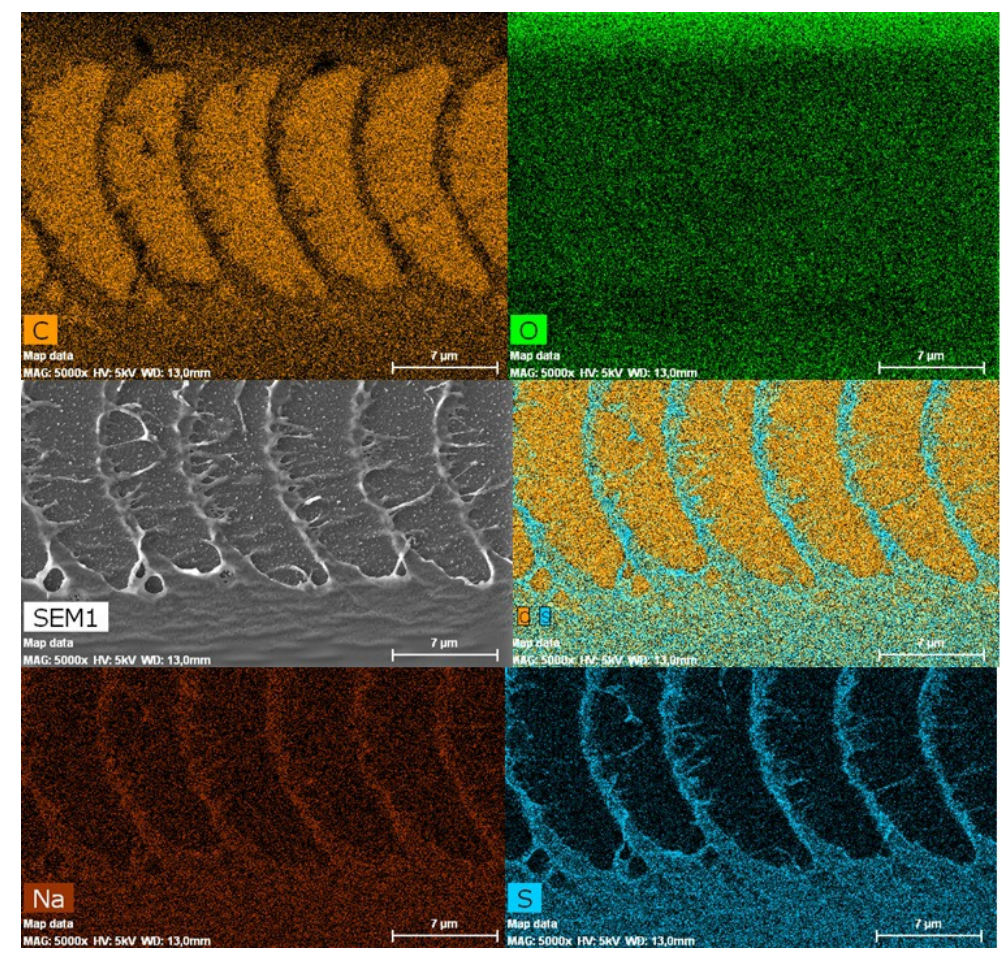

Figure 8 SEM micrograph and corresponding EDX elemental maps after picosecond laser ablation $(3.03 \mu \mathrm{m}) \mathrm{of} 140 \mathrm{~nm}$ PEDOT:PSS on PET, at $100 \mathrm{~mm} / \mathrm{s}$, pulse energy $\sim 3 \mu \mathrm{J}$, spot diameter in the focal plane of $12 \mu \mathrm{m}$.

Nevertheless, as can be seen from the EDX elemental mapping that a selective removal of PEDOT-PSS on PET was possible at $3.03 \mu \mathrm{m}$.

Other materials like DCV4T-Et2 and HDR014 were also ablated in this pulse regime. Because of the overlap on absorption spectra with PET, it is difficult to find a clear selective absorption band in the accessible wavelengths range of our system. The experiments were carried out at 3.31 and $\mu \mathrm{m} 3.37 \mu \mathrm{m}$ wavelengths. The experiments confirmed a dependence on the wavelength absorption coefficient on the ablation thresholds and showed the possibility to obtain clean grooves at low scanning speeds. The results obtained after picosecond laser ablation are illustrated in Figure 9 (DCV4T-Et2) and Figure 10 (HDR014).

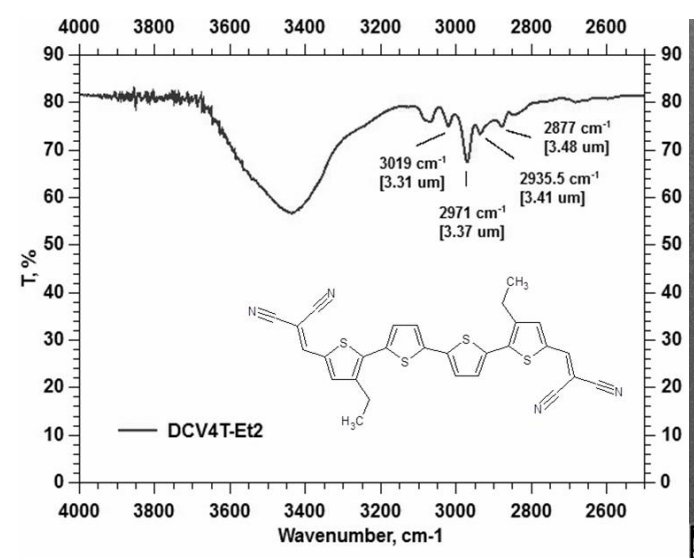

(a)

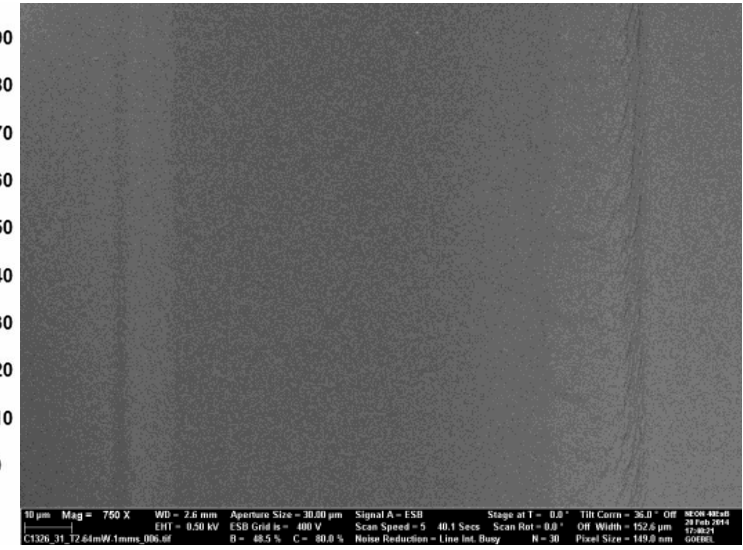

(b)

Figure 9 Picosecond laser patterning of DCV4T-Et2 on PET foil (a) Absorption spectrum of DCV4T-Et2 (b) SEM micrograph (composition mode) at $3.31 \mu \mathrm{m}, 1 \mathrm{~mm} / \mathrm{s}, 3.2 \mu \mathrm{J}$. 


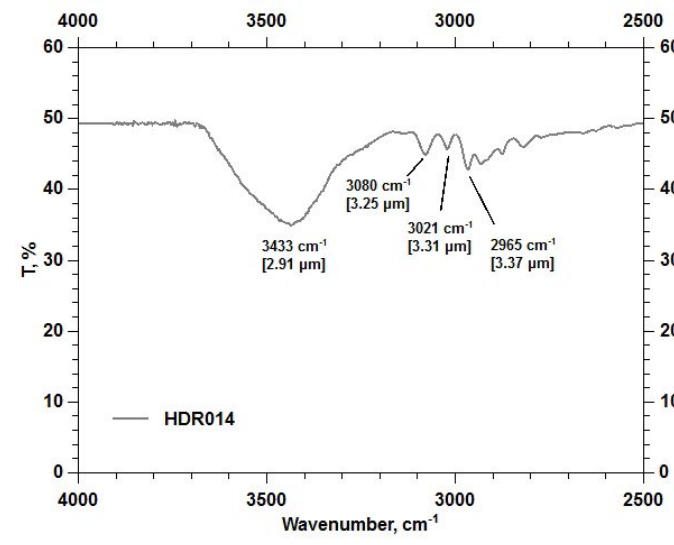

(a)

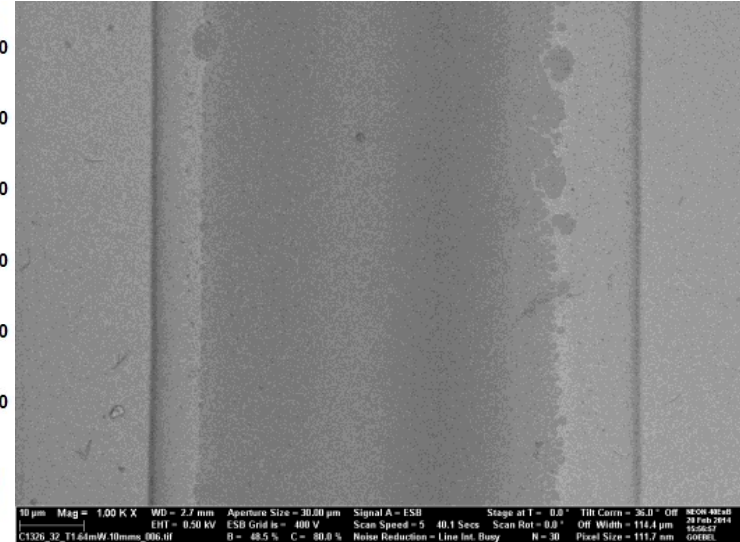

(b)

Figure 10 Picosecond laser patterning of HDR014 on PET foil (a) Absorption spectrum of HDR014 (b) SEM micrograph (composition mode) at $3.37 \mu \mathrm{m}, 10 \mathrm{~mm} / \mathrm{s}, 2.5 \mu \mathrm{J}$.

\subsection{BPAPF, $\alpha$ NPB and HDR014 nanosecond laser patterning}

In a similar fashion, experiments were carried out for BPAPF (140 nm thickness) on a glass substrate at $3.30 \mu \mathrm{m}$ (resonant peak) and at $3.40 \mu \mathrm{m}$ (non-resonant) wavelengths. At $3.30 \mu \mathrm{m}$ resonant absorption, processing was carried out with $170 \mathrm{~mW}$ power, a repetition rate of $20 \mathrm{kHz}$ and different scan speeds. The optical absorption spectrum of BPAPF and the result obtained at $3.30 \mu \mathrm{m}$ wavelength are illustrated in Figure 11.

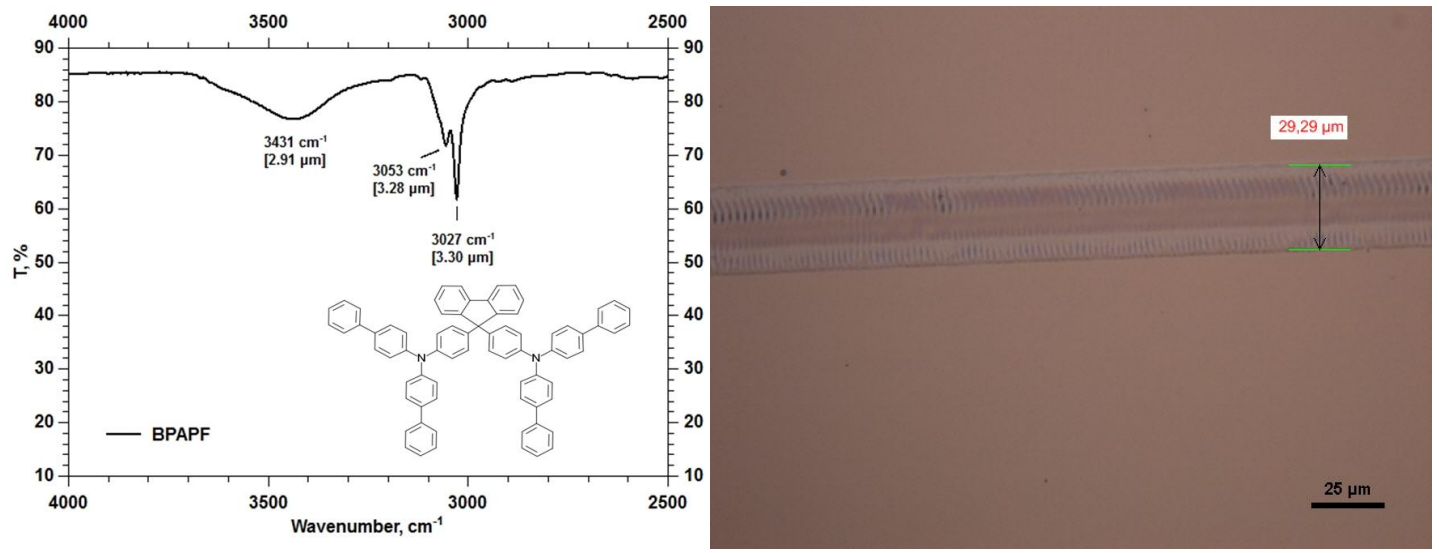

Figure 11 Absorption spectrum of BPAPF (left) and optical microscopic image obtained at $3.3 \mu \mathrm{m}$ resonant peak (right).

These observations show a clean debris-free ablated track at the central part, however at the edges there are pronounced higher rims. The possible reason for this kind of behavior at edges can be the melting of material due to nanosecond laser pulse, which is followed by re-solidification of material. Depth measured at the central part of the ablated groove by noncontact optical profiler is about $130 \mathrm{~nm}$. Thereafter, experiments for BPAPF at non-resonant wavelength $(3.40 \mu \mathrm{m})$ have been carried out with the identical laser processing parameters. Interestingly, there was no laser beam interaction with the thin film of BPAPF as there was no material removal and the surface topography remained intact.

Nanosecond laser experiments were continued for another thin organic film $\alpha \mathrm{NPB}$ at resonant and non-resonant wavelengths of absorption. As in the previous studies of PEDOT:PSS and BPAPF, a thickness of the $140 \mathrm{~nm} \alpha \mathrm{NPB}$ thin film on a glass substrate has been investigated. As can be seen from the absorption spectrum of $\alpha$ NPB (see Figure 12(a)), there is a peak of absorption at $3.30 \mu \mathrm{m}$ which was used for resonant ablation. These experiments were conducted at a 
power of $160 \mathrm{~mW}, 20 \mathrm{kHz}$ pulse repetition rate and $3.30 \mu \mathrm{m}$ wavelength. The results obtained in this case are illustrated with SEM micrograph in Figure 12(b).

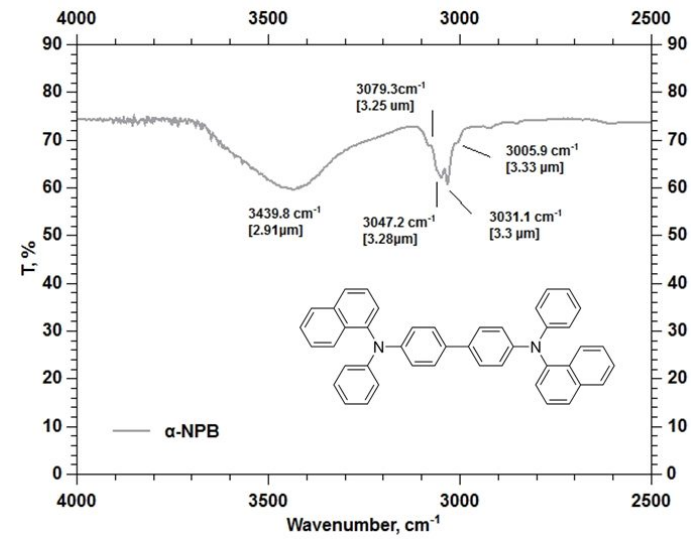

(a)

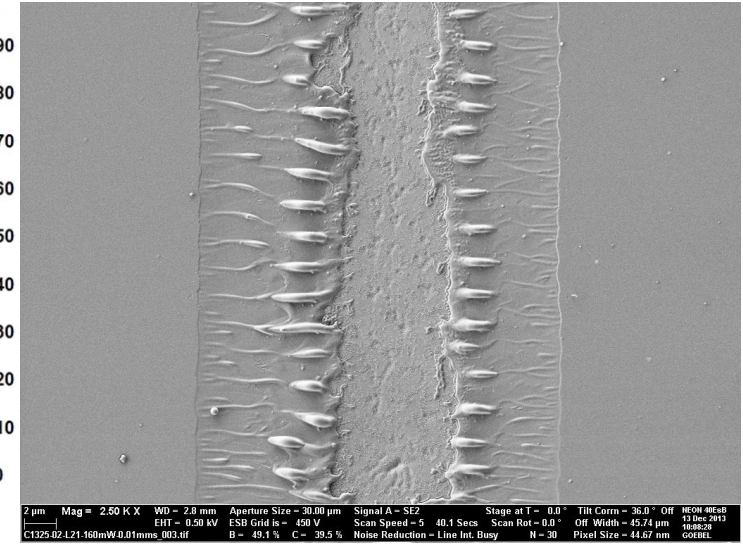

(b)

Figure 12 (a) Absorption spectrum of $\alpha$ NPB, indicating a resonant peak at $3.30 \mu \mathrm{m}$ and (b) laser patterned SEM micrograph of $\alpha$ NPB on glass substrate.

If the SEM micrograph is considered, it is inferred that at the central part of the ablated groove the material has been removed for the mostly. As far as the edges are concerned in SEM micrograph, it corresponds to the partially removed material which has been re-deposited after ablation process. At the non-resonant wavelength of $3.40 \mu \mathrm{m}$, there has not been any interaction of laser beam with the thin film of $\alpha$ NPB.

Finally, the experiments were carried out with nanosecond laser for the thin film of HDR014 on glass substrate. Investigations were conducted for identical thickness of $140 \mathrm{~nm}$ thin film of HDR014 on glass substrate. The processing is carried out with a power of $160 \mathrm{~mW}, 20 \mathrm{kHz}$ pulse repetition rate, different scan speeds, wavelengths of $3.37 \mu \mathrm{m}$ (resonant absorption) and $3.30 \mu \mathrm{m}$ (non-resonant). The optical absorption spectrum for HDR014 is shown in Figure 13 (a) and the result obtained at the wavelength of resonant absorption is shown in Figure 13 (b). The absorption spectrum of HDR014 clearly indicates a resonant peak of absorption at $3.37 \mu \mathrm{m}$. It can be seen in the Figure 13 (b) SEM micrograph that thin film removal is clean, however there is some roughness at the edges which is due to flakes after ablation.

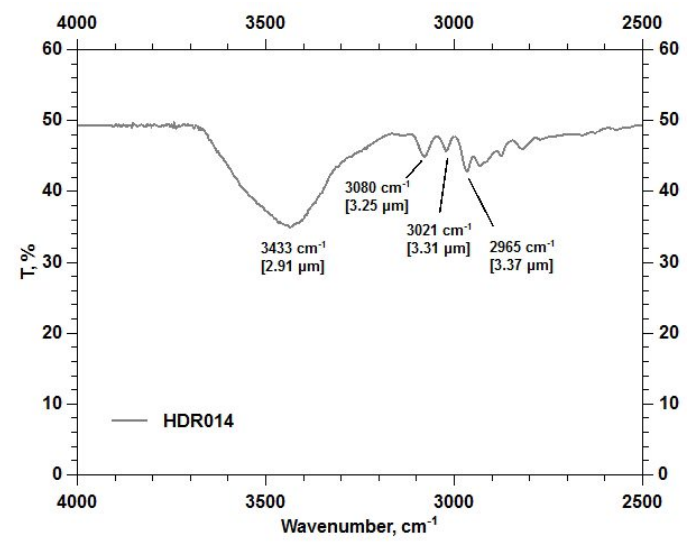

(a)

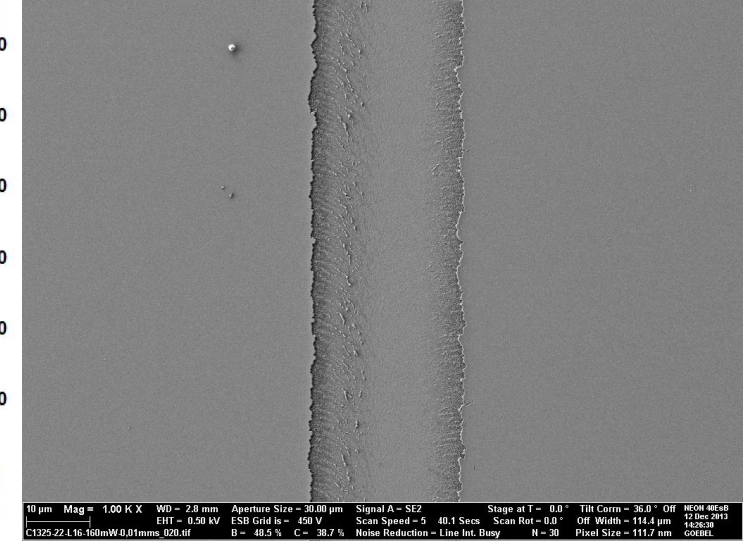

(b)

Figure 13 Absorption spectrum of HDR014 (a), and SEM micrograph of HDR014 thin film patterning on glass substrate at resonant wavelength $3.37 \mu \mathrm{m}(\mathrm{b})$.

For more detailed analysis of HDR014 patterned areas, EDS spectra of the ablated areas and non-ablated areas were investigated. The results of EDS analysis are depicted in Figure 14. 

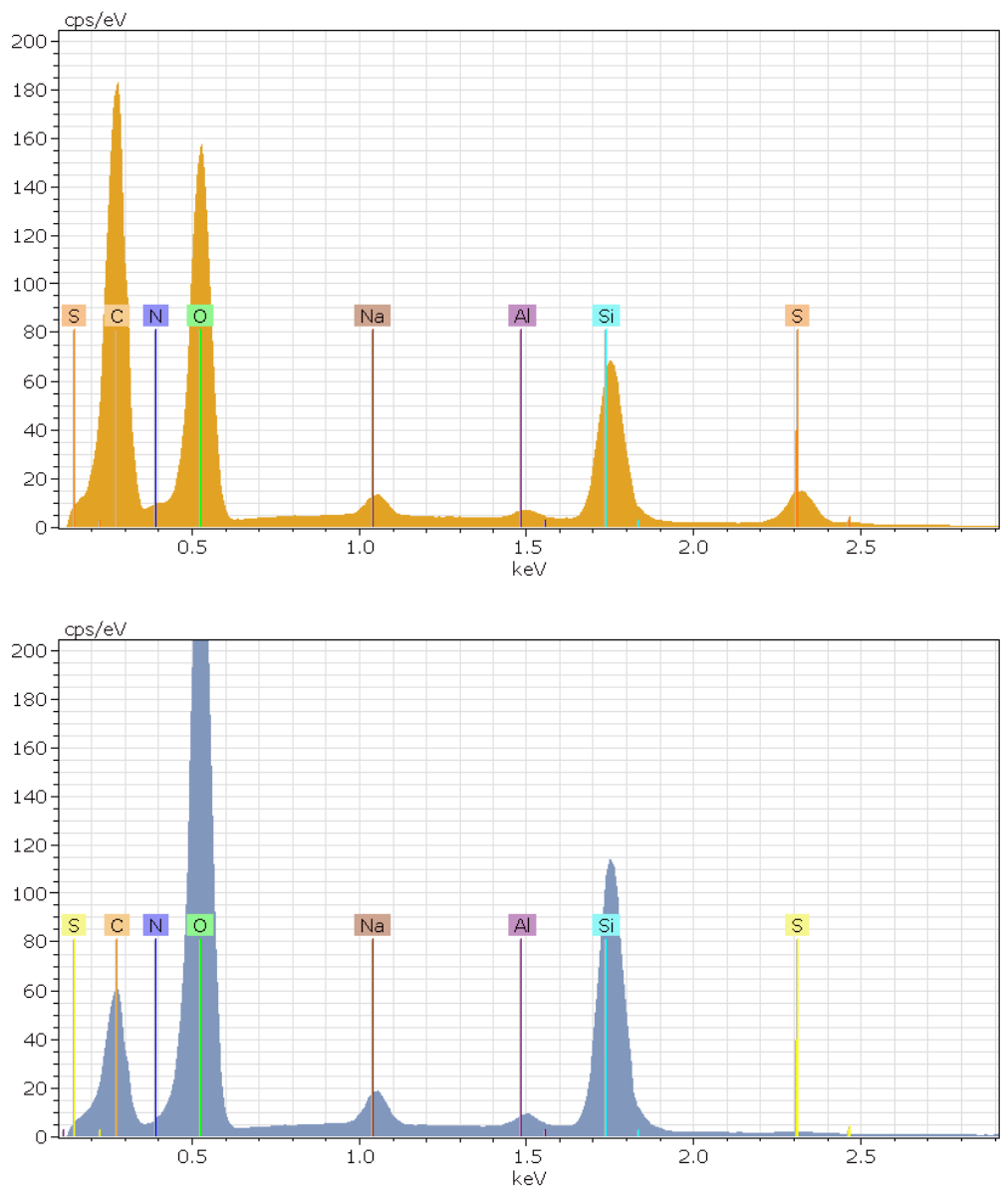

Figure 14 EDS spectra of HDR014 patterning on glass substrate, non-ablated area (top) and ablated area (bottom).

Looking at the EDS spectra as shown in Figure 14, it is observed that the Sulphur (S) peak has disappeared almost completely. This means that the oligothiophene material HDR014 has been removed almost completely from the glass substrate. Also the Carbon (C) content has been reduced widely in the ablated area (bottom) as compared to non-ablated area (top), and the remaining carbon may correspond to impurities. A more detailed investigation can be carried out by using Time-of-Flight Static Secondary Ion Mass Spectroscopy (TOF-S-SIMS)[14], as an analytical method to analyze the outermost molecular surface layer of the ablated area after thin films removal from the substrate.

\section{CONCLUSION}

We have applied the concept of RIA for selective patterning of the thin organic films. In these investigations, patterning of PEDOT:PSS on glass as well as on a PET foil, has been successfully demonstrated. Mid-IR selective ablation for DCV4T-Et2 on PET foil has been successful at lower scan speeds with picosecond laser. Moreover, a qualitative method for selective patterning of BPAPF, $\alpha \mathrm{NPB}$ and HDR014 thin organic films on glass substrate has been implemented. A detailed analysis with the help of optical microscope images, SEM micrographs, depth profiles and EDS spectra has been done. To summarize, it is possible to use RIA as a patterning scheme for thin organic films removal on a glass or foil substrate, however the scan speed has to be optimized for roll-to-roll processing as a final goal.

\section{FUTURE PERSPECTIVES}

It would be interesting to investigate and optimize the laser processes for other organic thin films (BPAPF, $\alpha$ NPB, HDR014) on foil (PET substrate) with mid-IR laser, which will definitely be a crucial step towards selective structuring the OPV layers in future. 


\section{ACKNOWLEDGEMENT}

We acknowledge the patience and care of Michael Göbel for evaluating a large number of laser scribes at the electron microscope. This work was conducted in the framework of the project "IMPROV", funded within the European Commission FP7 program. Part of the work was further supported by the German BMBF project "LOTSE" for developing high-efficiency OPV.

\section{REFERENCES}

[1] Logothetidis, S., "Flexible organic electronic devices: Materials, process and applications," Materials Science and Engineering: B, 152(1-3), 96-104 (2008).

[2] OE-A Roadmap for Organic and Printed Elecronicsm, 4th edition

[3] Kim, Y. H., Sachse, C., Machala, M. L., May, C., Müller-Meskamp, L. and Leo K., "Highly Conductive PEDOT:PSS Electrode with Optimized Solvent and Thermal Post-Treatment for ITO-Free Organic Solar Cells," Advanced Functional Materials, 21(6), 1076-1081 (2011).

[4] www.heliatek.com, press release January (2013).

[5] Yung, K. C., "Laser ablation of Upilex-S polyimide: influence of laser wavelength on chemical structure and composition in both ablated area and halo," Surface and Coatings Technology, 145 (1-3), 186-193 (2001).

[6] The European commission "IMPROV" project: http://www.fp7project-improv.eu (Website as viewed on $14 / 03 / 2014)$.

[7] Duering, M., Haglund, R. and Luther-Davies, B., "Resonant Infra-red Pulsed Laser Ablation of Polymers with single picosecond pulses generated by an optical parametric amplifier," Proc. Optical Society of America, Conference on Lasers and Electro-Optics (CLEO) and Quantum Electronics and Laser Science Conference (QELS), paper CMH3 (2010).

[8] Naithani, S., Grisard, A., Schaubroeck, D., Lallier, E. and Van Steenberge, G., Proc. International Congress on Laser Advanced Materials Processing (LAMP), 1-5 (2013).

[9] Guskova, O., Schuenemann, C., Eichhorn, K., Karsten, W., Levichkova, M., Grundmann, S. and Sommer, J. "Light Absorption in Organic Thin Films: The Importance of Oriented Molecules," Journal of Physical Chemistry C, 117(33), 17285-17293 (2013)

[10] Wynands, D., Levichkova, M., Riede, M., Pfeiffer, M., Baeuerle, P., Rentenberger, R., Denner, P. and Leo K., "Correlation between morphology and performance of low bandgap oligothiophene:C60 mixed heterojunctions in organic solar cells,” Journal of Applied Physics, 107, 014517 (2010).

[11] Aziz, H., Popovic, Z. D. and Hu, N., "Organic light emitting devices with enhanced operational stability at elevated temperatures", Applied Physics Letters, 81, 370 (2002).

[12] Myers, L. E., Bosenberg, W.R., Eckardt, R. C., Fejer, M. M. and R. L. Byer, "Multigrating quasi-phase-matched optical parametric oscillator in periodically poled LiNbO3," Optics Letters, 21, 591-593 (1996).

[13] Britton, P. E., Broderick, N. G. R., Richardson, D. J., Smith, P. G. R., Ross, G. W. and Hanna, D. C., "Wavelengthtunable high-power picosecond pulses from a fiber-pumped diode-seeded high-gain parametric amplifier," Optics Letters, 23, 1588-1590 (1998).

[14] Naithani, S., Schaubroeck, D., Vercammen, Y., Mandamparambil, R., Yakimets, I., Van Vaeck, L. and Van Steenberge G., "Excimer laser patterning of PEDOT:PSS thin-films on flexible barrier foils: A surface analysis study," Applied Surface Science 280, 504-511(2013). 